\title{
Regulation of Vessel Permeability by TRP Channels
}

\author{
Tullio Genova*t, Deborah Gagliotit and Luca Munaron \\ Department of Life Sciences and Systems Biology, University of Turin, Turin, Italy
}

The vascular endothelium constitutes a semi-permeable barrier between blood and interstitial fluids. Since an augmented endothelial permeability is often associated to pathological states, understanding the molecular basis for its regulation is a crucial biomedical and clinical challenge. This review focuses on the processes controlling paracellular permeability that is the permeation of fluids between adjacent endothelial cells (ECs). Cytosolic calcium changes are often detected as early events preceding the alteration of the endothelial barrier (EB) function. For this reason, great interest has been devoted in the last decades to unveil the molecular mechanisms underlying calcium fluxes and their functional relationship with vessel permeability. Beyond the dicotomic classification between store-dependent and independent calcium entry

OPEN ACCESS

Edited by:

Francesco Moccia,

University of Pavia, Italy

Reviewed by:

Andrea Gerbino,

National Research Council, Italy Alexander Dietrich,

Ludwig Maximilian University

of Munich, Germany

*Correspondence:

Tullio Genova

tullio.genova@unito.it

tThese authors have contributed equally to this work

Specialty section: This article was submitted to

Vascular Physiology, a section of the journal

Frontiers in Physiology

Received: 09 January 2020 Accepted: 07 April 2020

Published: 05 May 2020

Citation:

Genova T, Gaglioti D and Munaron L (2020) Regulation of Vessel Permeability by TRP Channels. Front. Physiol. 11:421. doi: 10.3389/fphys.2020.00421 at the plasma membrane level, the search for the molecular components of the related calcium-permeable channels revealed a difficult task for intrinsic and technical limitations. The contribution of redundant channel-forming proteins including members of TRP superfamily and Orai1, together with the very complex intracellular modulatory pathways, displays a huge variability among tissues and along the vascular tree. Moreover, calcium-independent events could significantly concur to the regulation of vascular permeability in an intricate and fascinating multifactorial framework.

Keywords: TRP, endothelial cell, store-operated $\mathrm{Ca}^{2+}$ entry channels, permeability, vessel permeability, microvessel, TRPC

\section{FEATURES OF THE VASCULAR ENDOTHELIUM}

Vascular endothelium lines the intima of the blood vessels, forming a semi-permeable interface between blood and interstitial fluids. Macromolecules cross this barrier via endo/exocytosis (transcellular permeability), while fluids and small solutes can pass endothelium through the space between adjacent ECs (paracellular permeability) (Komarova and Malik, 2010). The permeability of the endothelial layer is finely modulated in order to properly answer local metabolic demands. When the endothelium loses its barrier function, tissue inflammation occurs (Mehta and Malik, 2006).

Abbreviations: $4 \alpha \mathrm{PDD}, 4 \alpha$-phorbol 12,13-didecanoate; AA, arachidonic acid; AJs, adherent junction complexes; EB, endothelial barrier; ECM, extracellular matrix; ECs, endothelial cells; EET, 14,15-epoxyeicosatrienoic acid; FACs, focal adhesion complexes; hMEC, human microvascular endothelial cells; IEJs, intercellular endothelial junctions; LMECs, lung microvascular EC; MLC, Myosin light chain; MMP, matrix metalloproteinases; nSOCs, non-store-operated channels; ROS, reactive oxygen species; rPAECs, rat pulmonary aortic endothelial cells; rPMECs, rat pulmonary microvascular endothelial cells; SOCE, Store-Operated Calcium Entry; SOCs, store-operated cation channels; SP1, sphingosine-1-phosphate; SPHK1, sphingosine kinase 1; TER, transendothelial electrical resistance; TNF- $\alpha$, tumour necrosis factor- $\alpha$; TRP, Transient receptor potential. 
Responsible for paracellular permeability regulation are the intercellular contacts between ECs maintained by AJs, and the cellular adhesion to the underlying matrix through FACs (Cioffi and Stevens, 2006; Minshall and Malik, 2006). An enhanced endothelial permeability is the result of loss of contact between adjacent microvascular ECs and weakening of their adhesion to the ECM (Minshall and Malik, 2006). Vessel permeability is under the control of pro-inflammatory, thrombogenic mediators and growth factors binding to selective endothelial receptors. A convergent downstream effect is usually an increase of the cytosolic calcium concentration that precedes the enhancement of endothelial permeability. Indeed, cytosolic $\mathrm{Ca}^{2+}$ transients induce the retraction of the cell borders by weakening IEJs and cell-matrix junctions. Thus, while ECs change their morphology from a flattened to a rounded shape, gaps between adjacent ECs are formed, allowing the unrestricted flux of plasma proteins, including albumin, and liquid through IEJs (Lum and Malik, 1994; Mehta and Malik, 2006; Minshall and Malik, 2006).

Transient receptor potential channels play a key role in endothelial calcium fluxes occurring during physiological events and human diseases (Hsu et al., 2007; Watanabe et al., 2008; Banner et al., 2011; Galindo et al., 2018; Tsagareli and Nozadze, 2019). Transient receptor potential channels (TRPs) contribute to the regulation of the $\mathrm{EB}$, but the intracellular mechanisms are still partially elusive and highly variable in tissues as well as along the vascular tree.

Here we discuss the state of the art in the field, highlighting the discrepancies and conflicting evidence.

\section{ENDOTHELIAL PERMEABILITY ALONG THE VASCULAR TREE}

Since an increased endothelial permeability is considered a hallmark of vessel growth in many diseases (Bates and Harper, 2002), huge effort is devoted to uncover the underlying mechanisms, especially within the microcirculation.

In resting rat lung, the vascular filtration coefficient $\left(K_{f c}\right)$, indicative of the liquid flux, is near $42 \%$ in microvessels and just $19 \%$ in arterial regions (Parker and Yoshikawa, 2002). Similar results were found in canine lung (Mitzner and Robotham, 1979).

Such a striking difference can be ascribed to the density of fenestrae (Aird, 2007), together with EC shape, thickness and the protein composition of the IEJs. Tight junctions are well developed in large vessels, which have a conduct function, while they are weakened along capillaries, the canonical site for exchange with the surrounding tissue. Thus, the number and complexity of tight junctions appear to be inversely related to permeability (Aird, 2007). Microvascular ECs also express a greater amount of proteins involved in the interaction with the ECM (Chi et al., 2003), explaining why the ECM contribution to permeability prevails in capillaries (Qiao et al., 1995). In addition to these intrinsic mechanisms, also extrinsic factor contribute: blood flow and the relative mechanical stress is pulsatile in large vessels, while linear within capillaries (Mehta and Malik, 2006;
Sukriti et al., 2014). Finally, capillary permeability is strongly influenced by the coverage by pericytes, contractile cells wrapped around ECs (Attwell et al., 2016). Indeed, pericytes contraction reversibly opens endothelial gaps, while their loss irreversibly compromises EB (Edelman et al., 2006). Moreover, pericytes control tight junction expression and alignment (Winkler et al., 2012).

\section{CALCIUM SIGNALING REGULATES ENDOTHELIAL VESSEL PERMEABILITY}

Calcium signaling has a central role in the modulation of both physiological and pathological permeability (Curry, 1992; Bates and Curry, 1997; Kelly et al., 1998; Van Nieuw Amerongen et al., 1998; Bates and Harper, 2002; Minshall and Malik, 2006; De Bock et al., 2012). The intracellular calcium concentration $\left(\left[\mathrm{Ca}^{2+}\right]_{i}\right)$ increases in inflammation, causing a reorganization of endothelial gaps (Moore et al., 1998; Mehta and Malik, 2006).

Calcium signals modulate endothelial permeability partly via an ubiquitous mode referred to as SOCE, a calcium influx dictated by the depletion of endoplasmic reticulum (ER) calcium stores. The protein STIM1 is located in ER membranes acting as sensor of $\mathrm{Ca}^{2+}$ levels in the lumen: upon ER depletion, it underlies a rearrangement to plasma-membrane-ER junctions, where activates SOCs, that include the pore forming protein Orail (Smyth et al., 2010) and members of the TRP channel superfamily (Cheng et al., 2013; Ambudkar et al., 2017).

Inflammatory mediators (e.g., thrombin and histamine) bind to plasma membrane $G$ protein-coupled receptors and trigger InsP3-dependent $\mathrm{Ca}^{2+}$ release from $\mathrm{ER}$ and the following SOCE. The calcium-mediated phosphorylation of MLC drives the formation of actomyosin contractile units and stress fibers, which exert force on the IEJs, weakening them (Dudek and Garcia, 2001; Sandoval et al., 2001; Birukova et al., 2004). In addition, PKC phosphorylates junctional linking proteins vinculin and talin in IEJs and FACs (Lum and Malik, 1994; Rebecchi and Pentyala, 2000; Rhee, 2001). The disassembly of cell-cell and cellmatrix contacts (PKC-mediated passive cell retraction) and the concomitant establishment of contractile units (MLCK-mediated active cell contraction) lead to ECs rounding as well as the formation of intercellular gaps and permeability enhancement.

Early studies highlighted a variable contribution of calcium signaling to vascular permeability between larger and smaller vessels. Kelly et al. (1998) showed that an increase of $\left[\mathrm{Ca}^{2+}\right]_{i}$ enough to promote permeability in rPAECs, failed to exert any effect on rPMECs, initially suggesting an apparent uncoupling of $\left[\mathrm{Ca}^{2+}\right]_{i}$ signaling pathways or dominant $\mathrm{Ca}^{2+}$-independent mechanisms in microvasculature. In vitro permeation studies showed that the phosphodiesterase-4 inhibitor Rolipram inhibits SOC in PAECs while revealing it in PMVECs, with consequent shift of the fluid leakage site from big vessels to the microcirculation. Thus, the intracellular events associated with SOCE appear to be site specific, according to the variability of the response to proinflammatory stimuli (Dudek and Garcia, 2001; Wu et al., 2005). 
More recently, nSOCs were proposed as major players in microvascular permeability (Alvarez et al., 2006; Cioffi et al., 2009; Komarova et al., 2017; Phuong et al., 2017). Activated following agonist stimulation and independently of store depletion, nSOCs regulate EB (Mehta and Malik, 2006).

\section{TRPS IN ENDOTHELIAL PERMEABILITY}

Transient receptor potential channels are a superfamily of ion channels, which regulate the plasma membrane permeability to cations in response to a broad range of stimuli. Importantly, 19 of the 28 mammalian TRP channel isoforms are expressed in vascular ECs. Among them, all of the TRPC; TRPV1, -V2, and -V4; all of the TRPM except -M5; and TRPP1 and -P2 (Yao and Garland, 2005; Kwan et al., 2007).

In the following paragraphs, research works that discuss the role of TRP channels in the regulation of EC permeability will be discussed.

The involvement of TRPs in EB regulation is well established (Groschner et al., 1998; Owsianik et al., 2006; Ramsey et al., 2006; Tiruppathi et al., 2006; Moccia, 2012; Ong et al., 2016). TRPs can be activated by both intra- and extra-cellular messengers, as well as by physical or mechanical stimuli, promoting calcium signals and membrane depolarization, that may respectively, recruit store-operated and voltage-gated channels (Nilius, 2007; Mulier et al., 2017).

Consistent literature supports the involvement of TRP channels in both SOCE and non-SOCE calcium entry in ECs, as well as in endothelial permeability.

The molecular identity of the channels involved in endothelial SOCE gave rise to a long and exciting debate, with conflicting data due to methodological issues as culture conditions, overexpression systems, electrophysiological protocols, and pharmacological approaches (Groschner et al., 1998; Abdullaev et al., 2008; Trebak, 2009). The general accepted model recognizes highly $\mathrm{Ca}^{2+}$ selective SOCE currents through Orail channels and non-selective SOCE currents (the canonical $\mathrm{I}_{S O C}$ ) mediated by Orail and TRPC1, considered the predominant isoform expressed in human vascular endothelium (Tiruppathi et al., 2006; Worley et al., 2007; Cheng et al., 2013; Sabourin et al., 2015; Ambudkar et al., 2017; Lopez et al., 2020). The ER Ca ${ }^{2+}$ sensor STIM1 regulates both these kinds of channels (Lopez et al., 2020) and triggers Orail and TRPC1 activation by distinct C-terminus domains. Therefore, TRPC1 function is not only dependent on STIM1, but also requires the interaction with Orail (Ong et al., 2016). Moreover, Orail-mediated $\mathrm{Ca}^{2+}$ entry is needed for recruitment of TRPC1 and its insertion into membranes, while STIM1 gates the channel (Cheng et al., 2013). In human ECs, the phosphorylation of TRPC1 by PKCa is essential for the thrombin-induced activation of SOCE (Ahmmed et al., 2004). The expression of TRPC1 is also regulated: in human PAECs (hPAECs), the inflammatory cytokine TNF- $\alpha$ promotes TRPC1 overexpression (Paria et al., 2004) that triggers $\mathrm{Ca}^{2+}$ influx and enhances endothelial permeability (Tiruppathi et al., 2006). Upon thrombin exposure, RhoA triggers the association of TRPC1 to InsP3R, its translocation to the plasma membrane, calcium entry enhancement and finally the increase in endothelial permeability (Mehta et al., 2003). A role for SPHK1 was suggested in the pathway by which TRPC1-mediated calcium entry destabilizes AJs: TRPC1 holds SPHK1 constitutively in a suppressed state to prevent SP1 production, enabling inflammatory agonists to mediate vascular leak (Tauseef et al., 2016; Simmons et al., 2019).

TRPC1 overexpression in hMEC caused a twofold increase in thrombin-induced calcium depletion and in InsP3 store-operated cationic current. Actin-stress fiber formation was augmented and TER decreased (Paria et al., 2004). On the contrary, TRPC1 depletion reduced the global cytosolic $\mathrm{Ca}^{2+}$ response by $25 \%$ and $I_{S O C}$ by $50 \%$ (Brough et al., 2001). Further, the application of a specific antibody directed against an extracellular epitope of TRPC1 blocked thrombin- or $\mathrm{InsP}_{3}$-induced $\mathrm{Ca}^{2+}$ entry (Ahmmed et al., 2004).

Some members of TRPC family (i.e., TRPC-3, -6, and 7) can be stimulated by the membrane-permeant analog of DAG, 1-oleoyl-2-acetyl-sn-glycerol (Hofmann et al., 1999). Furthermore, these three channels show different levels of store-dependence: TRPC-3 is quite sensitive to InsP3-mediated responses, whereas TRPC-6 and -7 appear to be completely store-independent. Nevertheless, the independence of TRPC3 from InsP3 receptor activation has been demonstrated in that PLC activation in InsP3 receptor-deficient cell lines still retained TRPC-3 activation (Tiruppathi et al., 2002; Pocock et al., 2004).

Beside TRPC1, TRPC4 was also proposed as a major contributor in SOCE (Wu et al., 2005; Trebak, 2009; Sundivakkam et al., 2012; Antigny et al., 2017). TRPC1 and TRPC4 can heterodimerize in ECs, forming a single functional channel (Antoniotti et al., 2006; Ma et al., 2011; Du et al., 2014; Greenberg et al., 2019). TRPC4 binds to STIM1 (Worley et al., 2007) as well as to Orail: interestingly, Orail knockdown decreases the opening probability and the selectivity of TRPC1/4 channel (Cioffi et al., 2012; Thakore and Earley, 2019).

Knock out models for TRPC4 confirmed its role in permeability in vivo. $\mathrm{Ca}^{2+}$ influx evoked either by thrombin or a synthetic agonist (TFLLRNPNDK) was drastically diminished in isolated-perfused TRPC4-/- mouse lungs and in cultures ECs from the same model (Tiruppathi et al., 2002). This was associated with a lack of thrombin-induced actin-stress fiber formation and an impaired endothelial cell retraction.

Interestingly, TRPC4/5 channels can be mobilized not only via the Gq/11-protein-PLC pathway, but also following Gi/ocoupled signaling (Jeon et al., 2012).

Even if not demonstrated in ECs, recent evidences show that TRPC4/5 recruitment requires the dissociation of NHERF proteins from the channel $\mathrm{C}$ terminus, thus providing DAG sensitivity (Storch et al., 2017; Mederos y Schnitzler et al., 2018).

As stated above, TRPC6 may also be involved in EB dysfunction. Indeed, in frog mesenteric microvessels, VEGFinduced increase in vascular permeability can be mimicked by DAG, an agonist of TRPC3/6/7. Furthermore, flufenamic acid, which positively regulates TRPC6 but inhibits -C3 and -C7, enhances the effect exerted by VEGF (Pocock et al., 2004).

Evidences from studies with transfected cells demonstrated the TRPCs can mediate $\mathrm{Ca}^{2+}$ gated by DAG and are store and 
PKC independent (Nilius and Droogmans, 2001). Consistently, OAG-induced $\mathrm{Ca}^{2+}$ currents in TRPC-6-expressing cells were not sensitive to PKC inhibition, suggesting that TRPC-6 is directly gated by DAG (Hofmann et al., 1999).

Moreover, in Pocock et al. (2004) demonstrated in vivo that at least one mechanism of action of VEGF involves the increase in $\left[\mathrm{Ca}^{2+}\right]_{\mathrm{i}}$ through store-independent TRPC- 6 activation.

Finally, by the use of calcium imaging in isolated perfused rat lungs and patch clamp in rPAECs, TRPC6 was found to be critically involved in lung vascular leakage after stimulation with platelet-activating factor through its recruitment into caveolae (Samapati et al., 2012). In human PAECs, cell contraction exerted by thrombin is mediated by TRPC6 via a PKA-dependent pathway (Singh et al., 2007).

A member of TRPM subfamily, TRPM2, is highly expressed in ECs (Simmons et al., 2019), but its role in permeability is controversial. In cultured hPAECs, TRPM2 is opened by intracellular ADP-ribose and mediates the hyperpermeability triggered upon exposure to $\mathrm{H}_{2} \mathrm{O}_{2}$. Some in vivo studies reported that TRPM $2^{-/-}$mice develop pulmonary edema upon LPS treatment (Thomas et al., 2012), but other authors failed to reproduce the evidence (Hardaker et al., 2012). The discrepancy could be due to different mouse strains used in the experiments (Thakore and Earley, 2019).

In the ECs of blood vessels following spinal cord injury, TRPM4 resulted up-regulated, but the underlying mechanism in vascular permeability remains unclear (Grunewald et al., 2006).

Finally, some components of TRPV subfamily are under intense investigation.

The role of TRPV1 channels in this context is controversial. Indeed, Alvarez et al. demonstrated that the TRPV1 agonists did

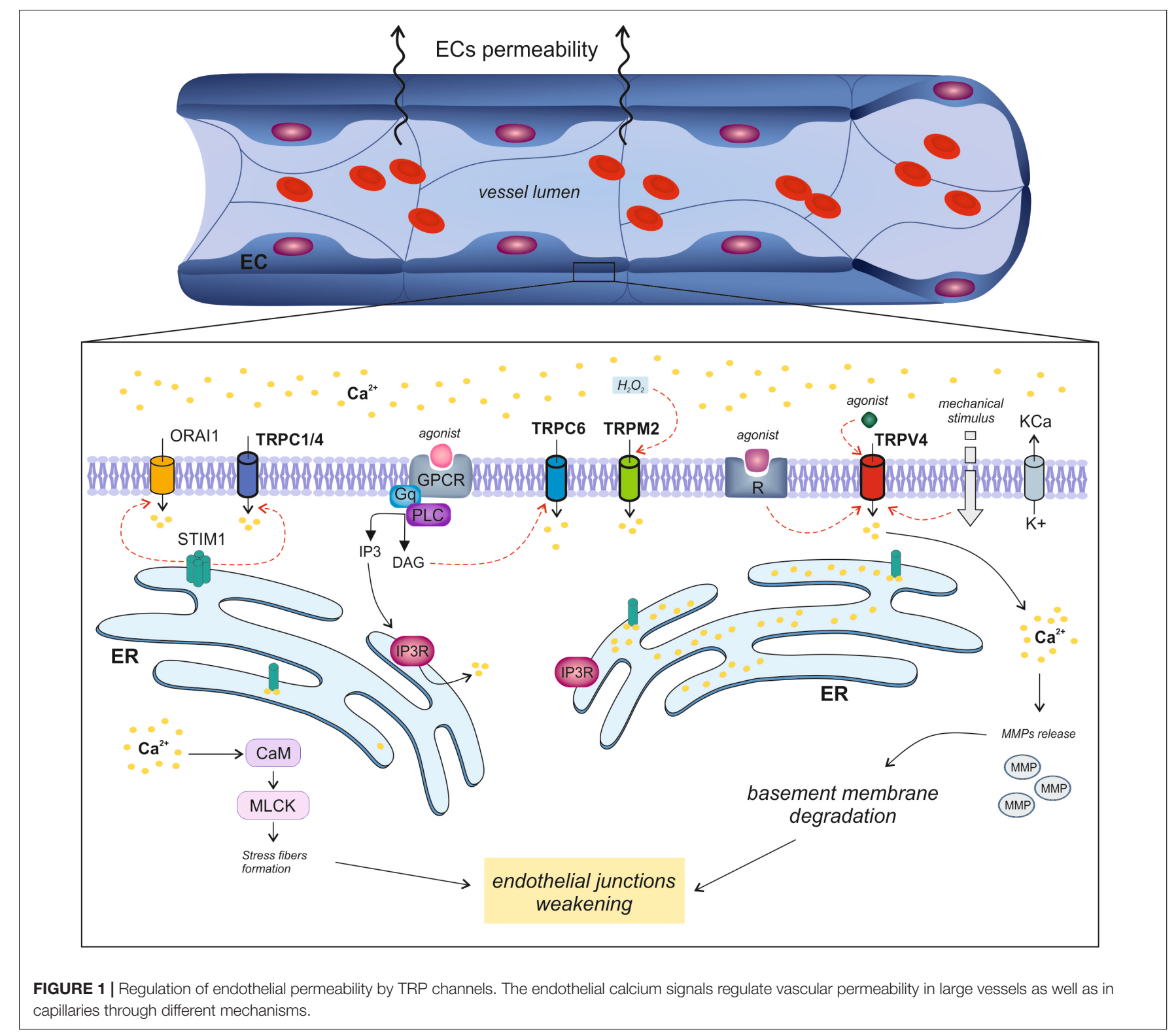


not affect lung permeability (Alvarez et al., 2005). Nevertheless, Wang et al. reported a protective effect by capsaicin, a potent TRPV1 activator, in rabbit lung ischemia-reperfusion injury (Wang et al., 2012; Thakore and Earley, 2019). On the other hand, TRPV1 expression is very low in hMECs (Thomas et al., 2012).

Nowadays, TRPV4 is attracting much attention for its influence on capillary barrier function (Morty and Kuebler, 2014; Simmons et al., 2019). Downregulation of TRPC1 and TRPC4 in artery and vein lung endothelium is associated with the loss of response to the SOCE-activating compound thapsigargin, supporting the idea that thapsigargin triggers a TRPC1/TRPC4-containing channel to increase permeability. However, permeability regulated by EET, a metabolite of the AA, was retained, opening the possibility for the involvement of other channels (Alvarez et al., 2005). 14,15-EET, which is produced upon high peak inspiratory pressure, activates TRPV4 and the subsequent increase in pulmonary vascular permeability (Watanabe et al., 2002; Hamanaka et al., 2007; Simmons et al., 2019; Thakore and Earley, 2019). TRPV4 is abundantly expressed in the endothelium from lung intra-alveolar capillaries and, to a lesser extent, from large, extra-alveolar vessels (Danbara et al., 1982; Alvarez et al., 2006). The TRPV4 agonist 4 $\alpha$ PDD increased permeability in wild type capillaries, with disruption of cell-matrix tethering, but failed to exert any effect in TRPV4 knockout mice; on the other hand, thapsigargin produced the same effect in extra-alveolar vessels (Alvarez et al., 2006; Thakore and Earley, 2019). Therefore, TRPC1 and TRPC4 appear to interfere preferentially with the lung extra-alveolar EB, whereas TRPV4 is prominently functional in lung capillaries (Cioffi et al., 2009). In mice, TRPV4 knockout prevented the permeability increase of lung microvasculature induced by both $4 \alpha \mathrm{PDD}$ and 14,15-EET, without affecting SOCE (Komarova et al., 2017). Moreover, TRPV4 triggers $\mathrm{Ca}^{2+}$-activated $\mathrm{K}^{+}$channels $(\mathrm{KCa})$ in rPMECs, enhancing the driving force for calcium entry and the response to TRPV4 (Lin et al., 2015). In mouse and human LMECs, ROS enhance vessel permeability via a Fyn Src kinaseTRPV4-dependent $\mathrm{Ca}^{2+}$ influx (Suresh et al., 2015; Thakore and Earley, 2019). TRPV4 seems to be also involved in the pulmonary edema associated with heart failure (Thorneloe et al., 2012). Interestingly, a NO/cGMP-dependent negative feedback loop was discovered for protection against the excessive microvascular barrier permeability (Yin et al., 2008). In LMECs, mechanically gated recruitment of TRPV4 elicits the release of the MMP MMP2 and MMP9, which degrade collagen IV and laminin, key structural components of the alveolar basement membrane, as well as integrins, intercellular E-cadherin and other intercellular targets (Villalta et al., 2014). The overall effect is the endothelial detachment from the basement membrane (Willette et al., 2008; Villalta and Townsley, 2013) and a permeability enhancement.

\section{REFERENCES}

Abdullaev, I. F., Bisaillon, J. M., Potier, M., Gonzalez, J. C., Motiani, R. K., and Trebak, M. (2008). Stiml and orail mediate crac currents and store-operated calcium entry important for endothelial cell proliferation. Circ. Res. 103, 1289-1299. doi: 10.1161/01.RES.0000338496.955 79.56
TRPV4 is also expressed in retinal hMECs and in intact blood vessels of the inner retina. Its selective agonist GSK101 promoted MEC permeability in association with disrupted F-actin organization, occludin downregulation and adherent contacts remodeling. Moreover, GSK101 increased the permeability of WT retinal blood vessels, but not in TRPV4 knockout mice, pointing to a major role for the channel in $\mathrm{Ca}^{2+}$ homeostasis and retinal barrier function (Phuong et al., 2017).

\section{CONCLUSION}

Calcium signaling tunes endothelial permeability in capillaries as well as in larger vessels. However, it is not surprising to find a huge variability in the related molecular machinery due to the well known heterogeneity of the endothelium in different tissues (Figure 1).

A general and established model for the relative contribution of SOCE and non-SOCE in macro- and micro-vessel permeability, as well as for the identity and function of TRP channels, is not available yet. A severe limitation is the lack of a detailed TRP proteomic pattern and intracellular targeting landscape in capillary and arterial/vein ECs. In addition, selective pharmacological compounds are only available for some TRP members and some of them are not suitable for systemic treatments due to their highly toxicity (Meotti et al., 2014; Rodrigues et al., 2016; Rubaiy et al., 2017; Lawhorn et al., 2020).

Finally, the simple detection of a variety of active calcium entry mechanisms, with their complex protein machineries, does not necessarily provide an evidence for their exclusive functional involvement in vessel permeability. Trebak and coworkers reported that the acute barrier disruption activated by thrombin in hUVECs and hMECs requires endoplasmicreticulum localized STIM1 independently of Orai1, MLCK, and $\mathrm{Ca}^{2+}$ entry across the plasma membrane. STIM1 couples the thrombin receptor, recruits guanosine triphosphatase RhoA and stimulates MLC phosphorylation, finally leading to formation of actin stress fibers and loss of cell-cell adhesion (Shinde et al., 2013; Stolwijk et al., 2016).

Vascular permeability is probably the result of a concurrence among diverse, tissue-dependent intracellular processes, all contributing in variable weights to the overall event.

\section{AUTHOR CONTRIBUTIONS}

All authors listed have made a substantial, direct and intellectual contribution to the work, and approved it for publication.

Ahmmed, G. U., Mehta, D., Vogel, S., Holinstat, M., Paria, B. C., Tiruppathi, C., et al. (2004). Protein kinase $\mathrm{C} \alpha$ phosphorylates the TRPC1 channel and regulates store-operated $\mathrm{Ca} 2+$ entry in endothelial cells. J. Biol. Chem. 279, 20941-20949. doi: 10.1074/jbc.M313975200

Aird, W. C. (2007). Phenotypic heterogeneity of the endothelium: I. Structure, function, and mechanisms. Circ. Res. 100, 158-173. doi: 10.1161/01.RES. $0000255691.76142 .4 \mathrm{a}$ 
Alvarez, D. F., King, J. A., and Townsley, M. I. (2005). Resistance to store depletion-induced endothelial injury in rat lung after chronic heart failure. Am. J. Respir. Crit. Care Med. 172, 1153-1160. doi: 10.1164/rccm.200506$847 \mathrm{OC}$

Alvarez, D. F., King, J. A., Weber, D., Addison, E., Liedtke, W., and Townsley, M. I. (2006). Transient receptor potential vanilloid 4-mediated disruption of the alveolar septal barrier: a novel mechanism of acute lung injury. Circ. Res. 99, 988-995. doi: 10.1161/01.RES.0000247065.11756.19

Ambudkar, I. S., de Souza, L. B., and Ong, H. L. (2017). TRPC1, Orail, and STIM1 in SOCE: friends in tight spaces. Cell Calcium 63, 33-39. doi: 10.1016/j.ceca. 2016.12.009

Antigny, F., Sabourin, J., Saüc, S., Bernheim, L., Koenig, S., and Frieden, M. (2017). TRPC1 and TRPC4 channels functionally interact with STIM1L to promote myogenesis and maintain fast repetitive $\mathrm{Ca} 2+$ release in human myotubes. Biochim. Biophys. Acta Mol. Cell Res. 1864, 806-813. doi: 10.1016/j.bbamcr. 2017.02.003

Antoniotti, S., Pla, A., Barral, S., Scalabrino, O., Munaron, L., et al. (2006). Interaction between TRPC channel subunits in endothelial cells. J. Recept. Signal Transduct. 26, 225-240. doi: 10.1080/10799890600784050

Attwell, D., Mishra, A., Hall, C. N., O'Farrell, F. M., and Dalkara, T. (2016). What is a pericyte? J. Cereb. Blood Flow Metab. 36, 451-455. doi: 10.1177/ 0271678X15610340

Banner, K. H., Igney, F., and Poll, C. (2011). TRP channels: emerging targets for respiratory disease. Pharmacol. Ther. 130, 371-384. doi: 10.1016/j.pharmthera. 2011.03.005

Bates, D. O., and Curry, F. E. (1997). Vascular endothelial growth factor increases microvascular permeability via a $\mathrm{Ca}(2+)$-dependent pathway. Am. J. Physiol. Circ. Physiol. 273, H687-H694. doi: 10.1152/ajpheart.1997.273.2.H687

Bates, D. O., and Harper, S. J. (2002). Regulation of vascular permeability by vascular endothelial growth factors. Vascul. Pharmacol. 39, 225-237. doi: 10. 1016/S1537-1891(03)00011-9

Birukova, A. A., Smurova, K., Birukov, K. G., Usatyuk, P., Liu, F., Kaibuchi, K., et al. (2004). Microtubule disassembly induces cytoskeletal remodeling and lung vascular barrier dysfunction: role of Rho-dependent mechanisms. J. Cell. Physiol. 201, 55-70. doi: 10.1002/jcp.20055

Brough, G. H., Wu, S., Cioffi, D., Moore, T. M., Li, M., Dean, N., et al. (2001). Contribution of endogenously expressed Trp1 to a Ca2+-selective, storeoperated Ca2+ entry pathway. FASEB J. 15, 1727-1738. doi: 10.1096/fj.010108com

Cheng, K. T., Ong, H. L., Liu, X., and Ambudkar, I. S. (2013). Contribution and regulation of TRPC channels in store-operated Ca2+ entry. Curr. Top. Membr. 71, 149-179. doi: 10.1016/B978-0-12-407870-3.00007-X

Chi, J., Chang, H. Y., Haraldsen, G., Jahnsen, F. L., Troyanskaya, O. G., Chang, D. S., et al. (2003). Endothelial cell diversity revealed by global expression profiling. Proc. Natl. Acad. Sci. U.S.A. 100, 10623-10628. doi: 10.1073/pnas. 1434429100

Cioffi, D. L., Lowe, K., Alvarez, D. F., Barry, C., and Stevens, T. (2009). TRPing on the lung endothelium: calcium channels that regulate barrier function. Antioxid. Redox Signal. 11, 765-776. doi: 10.1089/ars.2008.2221

Cioffi, D. L., and Stevens, T. (2006). Regulation of endothelial cell barrier function by store-operated calcium entry. Microcirculation 13, 709-723. doi: 10.1080/ 10739680600930354

Cioffi, D. L., Wu, S., Chen, H., Alexeyev, M., St Croix, C. M., Pitt, B. R., et al. (2012). Orail determines calcium selectivity of an endogenous TRPC heterotetramer channel. Circ. Res. 110, 1435-1444. doi: 10.1161/CIRCRESAHA.112.269506

Curry, F. E. (1992). Modulation of venular microvessel permeability by calcium influx into endothelial cells. FASEB J. 6, 2456-2466. doi: 10.1096/fasebj.6.7. 1563597

Danbara, T., Kasano, T., Owada, T., Natori, H., Arai, T., et al. (1982). Extra-alveolar vessels and intra-alveolar vessels: two cases of pulmonary arteriovenous fistula. Jpn. J. Thorac. Dis. 20, 230-238. doi: 10.11389/jjrs1963.20.230

De Bock, M., Culot, M., Wang, N., Da Costa, A., Decrock, E., Bol, M., et al. (2012). Low extracellular $\mathrm{Ca} 2+$ conditions induce an increase in brain endothelial permeability that involves intercellular Ca2+ waves. Brain Res. 1487, 78-87. doi: 10.1016/j.brainres.2012.06.046

Du, J., Ma, X., Shen, B., Huang, Y., Birnbaumer, L., et al. (2014). TRPV4, TRPC1, and TRPP2 assemble to form a flow-sensitive heteromeric channel. FASEB J. 28, 4677-4685. doi: 10.1096/fj.14-251652
Dudek, S. M., and Garcia, J. G. N. (2001). Cytoskeletal regulation of pulmonary vascular permeability. J. Appl. Physiol. 91, 1487-1500. doi: 10.1152/jappl.2001. 91.4.1487

Edelman, D. A., Jiang, Y., Tyburski, J., Wilson, R. F., and Steffes, C. (2006). Pericytes and their role in microvasculature homeostasis. J. Surg. Res. 135, 305-311. doi: 10.1016/j.jss.2006.06.010

Galindo, T., Reyna, J., and Weyer, A. (2018). Evidence for transient receptor potential (TRP) channel contribution to arthritis pain and pathogenesis. Pharmaceuticals 11:105. doi: 10.3390/ph11040105

Greenberg, H. Z. E., Carlton-Carew, S. R. E., Zargaran, A. K., Jahan, K. S., Birnbaumer, L., et al. (2019). Heteromeric TRPV4/TRPC1 channels mediate calcium-sensing receptor-induced relaxations and nitric oxide production in mesenteric arteries: comparative study using wild-type and TRPC1-/- mice. Channels 13, 410-423. doi: 10.1080/19336950.2019.1673131

Groschner, K., Hingel, S., Lintschinger, B., Balzer, M., Romanin, C., Zhu, X., et al. (1998). TRP proteins form store-operated cation channels in human vascular endothelial cells. FEBS Lett. 437, 101-106. doi: 10.1016/S0014-5793(98) 01212-5

Grunewald, M., Avraham, I., Dor, Y., Bachar-Lustig, E., Itin, A., Yung, S., et al. (2006). VEGF-induced adult neovascularization: recruitment, retention, and role of accessory cells. Cell 124, 175-189. doi: 10.1016/j.cell.2005.10.036

Hamanaka, K., Jian, M.-Y., Weber, D. S., Alvarez, D. F., Townsley, M. I., Al-Mehdi, A. B., et al. (2007). TRPV4 initiates the acute calcium-dependent permeability increase during ventilator-induced lung injury in isolated mouse lungs. Am. J. Physiol. Lung Cell. Mol. Physiol. 293, L923-L932. doi: 10.1152/ajplung.00221. 2007

Hardaker, L., Bahra, P., de Billy, B. C., Freeman, M., Kupfer, N., Wyss, D., et al. (2012). The ion channel transient receptor potential melastatin-2 does not play a role in inflammatory mouse models of chronic obstructive pulmonary diseases. Respir. Res. 13:30. doi: 10.1186/1465-9921-13-30

Hofmann, T., Obukhov, A. G., Schaefer, M., Harteneck, C., Gudermann, T., and Schultz, G. (1999). Direct activation of human TRPC6 and TRPC3 channels by diacylglycerol. Nature 397, 259-263. doi: 10.1038/16711

Hsu, Y. J., Hoenderop, J. G. J., and Bindels, R. J. M. (2007). TRP channels in kidney disease. Biochim. Biophys. Acta Mol. Basis Dis. 1772, 928-936. doi: 10.1016/j. bbadis.2007.02.001

Jeon, J. P., Hong, C., Park, E. J., Jeon, J. H., Cho, N. H., Kim, I. G., et al. (2012). Selective Gai subunits as novel direct activators of transient receptor potential canonical (TRPC) 4 and TRPC5 channels. J. Biol. Chem. 287, 17029-17039. doi: $10.1074 /$ jbc.M111.326553

Kelly, J. J., Moore, T. M., Babal, P., Diwan, A. H., Stevens, T., and Thompson, W. J. (1998). Pulmonary microvascular and macrovascular endothelial cells: differential regulation of Ca2+ and permeability. Am. J. Physiol. 274, L810L819. doi: 10.1152/ajplung.1998.274.5.L810

Komarova, Y., and Malik, A. B. (2010). Regulation of endothelial permeability via paracellular and transcellular transport pathways. Annu. Rev. Physiol. 72, 463-493. doi: 10.1146/annurev-physiol-021909-135833

Komarova, Y. A., Kruse, K., Mehta, D., and Malik, A. B. (2017). Protein interactions at endothelial junctions and signaling mechanisms regulating endothelial permeability. Circ. Res. 120, 179-206. doi: 10.1161/CIRCRESAHA.116.306534

Kwan, H. Y., Huang, Y., and Yao, X. (2007). TRP channels in endothelial function and dysfunction. Biochim. Biophys. Acta Mol. Basis Dis. 1772, 907-914. doi: 10.1016/j.bbadis.2007.02.013

Lawhorn, B. G., Brnardic, E. J., and Behm, D. J. (2020). Recent advances in TRPV4 agonists and antagonists. Bioorg. Med. Chem. Lett. 30:127022. doi: 10.1016/j. bmcl.2020.127022

Lin, M. T., Jian, M. Y., Taylor, M. S., Cioffi, D. L., Yap, F. C., Liedtke, W., et al. (2015). Functional coupling of TRPV4, IK, and SK channels contributes to Ca2+-dependent endothelial injury in rodent lung. Pulm. Circ. 5, 279-290. doi: $10.1086 / 680166$

Lopez, J. J., Jardin, I., Albarrán, L., Sanchez-Collado, J., Cantonero, C., Salido, G. M., et al. (2020). Molecular basis and regulation of store-operated calcium entry. Adv. Exp. Med. Biol. 1131, 445-469. doi: 10.1007/978-3-030-12457-1_17

Lum, H., and Malik, A. B. (1994). Regulation of vascular endothelial barrier function. Am. J. Physiol. Cell. Mol. Physiol. 267, L223-L241. doi: 10.1152/ ajplung.1994.267.3.L223

Ma, X., Cheng, K. T., Wong, C. O., O’Neil, R. G., Birnbaumer, L., Ambudkar, I. S., et al. (2011). Heteromeric TRPV4-C1 channels contribute to store-operated 
Ca2+ entry in vascular endothelial cells. Cell Calcium 50, 502-509. doi: 10.1016/ j.ceca.2011.08.006

Mederos y Schnitzler, M., Gudermann, T., and Storch, U. (2018). Emerging roles of diacylglycerol-sensitive TRPC4/5 channels. Cells 7:218. doi: 10.3390/ cells7110218

Mehta, D., Ahmmed, G. U., Paria, B. C., Holinstat, M., Voyno-Yasenetskaya, T., Tiruppathi, C., et al. (2003). RhoA interaction with inositol 1,4,5-trisphosphate receptor and transient receptor potential channel-1 regulates $\mathrm{Ca} 2+$ entry: role in signaling increased endothelial permeability. J. Biol. Chem. 278, 33492-33500. doi: 10.1074/jbc.M302401200

Mehta, D., and Malik, A. B. (2006). Signaling mechanisms regulating endothelial permeability. Physiol. Rev. 86, 279-367. doi: 10.1152/physrev.00012.2005

Meotti, F. C., De Andrade, E. L., and Calixto, J. B. (2014). TRP modulation by natural compounds. Handb. Exp. Pharmacol. 223, 1177-1238. doi: 10.1007/9783-319-05161-1_19

Minshall, R. D., and Malik, A. B. (2006). Transport across the endothelium: regulation of endothelial permeability. Handb. Exp. Pharmacol. 176, 107-144. doi: 10.1007/3-540-32967-6-4

Mitzner, W., and Robotham, J. L. (1979). Distribution of interstitial compliance and filtration coefficient in canine lung. Lymphology 12, 140-148.

Moccia, F. (2012). Update on vascular endothelial Ca 2+ signalling: a tale of ion channels, pumps and transporters. World J. Biol. Chem. 3, 127-158. doi: 10.4331/wjbc.v3.i7.127

Moore, T. M., Chetham, P. M., Kelly, J. J., and Stevens, T. (1998). Signal transduction and regulation of lung endothelial cell permeability. Interaction between calcium and cAMP. Am. J. Physiol. Cell. Mol. Physiol. 275, L203-L222. doi: 10.1152/ajplung.1998.275.2.L203

Morty, R. E., and Kuebler, W. M. (2014). TRPV4: an exciting new target to promote alveolocapillary barrier function. Am. J. Physiol. Lung Cell. Mol. Physiol. 307, L817-L821. doi: 10.1152/ajplung.00254.2014

Mulier, M., Vriens, J., and Voets, T. (2017). TRP channel pores and local calcium signals. Cell Calcium 66, 19-24. doi: 10.1016/j.ceca.2017.04.007

Nilius, B. (2007). TRP channels in disease. Biochim. Biophys. Acta 1772, 805-812. doi: 10.1016/j.bbadis.2007.02.002

Nilius, B., and Droogmans, G. (2001). Ion channels and their functional role in vascular endothelium. Physiol. Rev. 81, 1415-1459. doi: 10.1152/physrev.2001. 81.4.1415

Ong, H. L., De Souza, L. B., and Ambudkar, I. S. (2016). Role of TRPC channels in store-operated calcium entry. Adv. Exp. Med. Biol. 898, 87-109. doi: 10.1007/ 978-3-319-26974-0_5

Owsianik, G., Talavera, K., Voets, T., and Nilius, B. (2006). Permeation and selectivity of TRP channels. Annu. Rev. Physiol. 68, 685-717. doi: 10.1146/ annurev.physiol.68.040204.101406

Paria, B. C., Vogel, S. M., Ahmmed, G. U., Alamgir, S., Shroff, J., Malik, A. B., et al. (2004). Tumor necrosis factor- $\alpha$-induced TRPC1 expression amplifies storeoperated Ca $2+$ influx and endothelial permeability. Am. J. Physiol. Cell. Mol. Physiol. 287, L1303-L1313. doi: 10.1152/ajplung.00240.2004

Parker, J. C., and Yoshikawa, S. (2002). Vascular segmental permeabilities at high peak inflation pressure in isolated rat lungs. Am. J. Physiol. Cell. Mol. Physiol. 283, L1203-L1209. doi: 10.1152/ajplung.00488.2001

Phuong, T. T. T., Redmon, S. N., Yarishkin, O., Winter, J. M., Li, D. Y., and Križaj, D. (2017). Calcium influx through TRPV4 channels modulates the adherens contacts between retinal microvascular endothelial cells. J. Physiol. 595, 6869-6885. doi: 10.1113/JP275052

Pocock, T. M., Foster, R. R., and Bates, D. O. (2004). Evidence of a role for TRPC channels in VEGF-mediated increased vascular permeability in vivo. Am. J. Physiol. Hear. Circ. Physiol. 286, H1015-H1026. doi: 10.1152/ajpheart.00826. 2003

Qiao, R. L., Wang, H. S., Yan, W., Odekon, L. E., Del Vecchio, P. J., Smith, T. J., et al. (1995). Extracellular matrix hyaluronan is a determinant of the endothelial barrier. Am. J. Physiol. 269, C103-C109. doi: 10.1152/ajpcell.1995.269. 1.C103

Ramsey, I. S., Delling, M., and Clapham, D. E. (2006). An introduction to TRP channels. Annu. Rev. Physiol. 68, 619-647. doi: 10.1146/annurev.physiol.68. 040204.100431

Rebecchi, M. J., and Pentyala, S. N. (2000). Structure, function, and control of phosphoinositide-specific phospholipase C. Physiol. Rev. 80, 1291-1335. doi: 10.1152/physrev.2000.80.4.1291
Rhee, S. G. (2001). Regulation of phosphoinositide-specific phospholipase C. Annu. Rev. Biochem. 70, 281-312. doi: 10.1146/annurev.biochem.70.1.281

Rodrigues, T., Sieglitz, F., and Bernardes, G. J. L. (2016). Natural product modulators of transient receptor potential (TRP) channels as potential anticancer agents. Chem. Soc. Rev. 45, 6130-6137. doi: 10.1039/c5cs00916b

Rubaiy, H. N., Ludlow, M. J., Bon, R. S., and Beech, D. J. (2017). Pico145 powerful new tool for TRPC1/4/5 channels. Channels 11,362-364. doi: 10.1080/ 19336950.2017.1317485

Sabourin, J., Le Gal, L., Saurwein, L., Haefliger, J. A., Raddatz, E., and Allagnat, F. (2015). Store-operated Ca2+ entry mediated by Orail and TRPC1 participates to insulin secretion in rat $\beta$-cells. J. Biol. Chem. 290, 30530-30539. doi: 10.1074/ jbc.M115.682583

Samapati, R., Yang, Y., Yin, J., Stoerger, C., Arenz, C., Dietrich, A., et al. (2012). Lung endothelial $\mathrm{Ca} 2+$ and permeability response to platelet-activating factor is mediated by acid sphingomyelinase and transient receptor potential classical 6 . Am. J. Respir. Crit. Care Med. 185, 160-170. doi: 10.1164/rccm.201104-0717OC

Sandoval, R., Malik, A. B., Minshall, R. D., Kouklis, P., Ellis, C. A., et al. (2001). $\mathrm{Ca} 2+$ signalling and $\mathrm{PKC} \alpha$ activate increased endothelial permeability by disassembly of VE-cadherin junctions. J. Physiol. 533, 433-445. doi: 10.1111/ j.1469-7793.2001.0433a.x

Shinde, A. V., Motiani, R. K., Zhang, X., Abdullaev, I. F., Adam, A. P., GonzálezCobos, J. C., et al. (2013). STIM1 controls endothelial barrier function independently of Orail and Ca2+ entry. Sci. Signal. 6:ra18. doi: 10.1126/ scisignal.2003425

Simmons, S., Erfinanda, L., Bartz, C., and Kuebler, W. M. (2019). Novel mechanisms regulating endothelial barrier function in the pulmonary microcirculation. J. Physiol. 597, 997-1021. doi: 10.1113/JP276245

Singh, I., Knezevic, N., Ahmmed, G. U., Kini, V., Malik, A. B., and Mehta, D. (2007). Galphaq-TRPC6-mediated Ca2+ entry induces RhoA activation and resultant endothelial cell shape change in response to thrombin. J. Biol. Chem. 282, 7833-7843. doi: 10.1074/jbc.M608288200

Smyth, J. T., Hwang, S.-Y., Tomita, T., DeHaven, W. I., Mercer, J. C., and Putney, J. W. (2010). Activation and regulation of store-operated calcium entry. J. Cell. Mol. Med. 14, 2337-2349. doi: 10.1111/j.1582-4934.2010.01168.x

Stolwijk, J. A., Zhang, X., Gueguinou, M., Zhang, W., Matrougui, K., Renken, C., et al. (2016). Calcium signaling is dispensable for receptor regulation of endothelial barrier function. J. Biol. Chem. 291, 22894-22912. doi: 10.1074/jbc. M116.756114

Storch, U., Forst, A. L., Pardatscher, F., Erdogmus, S., Philipp, M., Gregoritza, M., et al. (2017). Dynamic NHERF interaction with TRPC4/5 proteins is required for channel gating by diacylglycerol. Proc. Natl. Acad. Sci. U.S.A. 114, E37-E46. doi: 10.1073/pnas.1612263114

Sukriti, S., Tauseef, M., Yazbeck, P., and Mehta, D. (2014). Mechanisms regulating endothelial permeability. Pulm. Circ. 4, 535-551. doi: 10.1086/677356

Sundivakkam, P. C., Freichel, M., Singh, V., Yuan, J. P., Vogel, S. M., Flockerzi, V., et al. (2012). The Ca2+ sensor stromal interaction molecule 1 (STIM1) is necessary and sufficient for the store-operated $\mathrm{Ca} 2+$ entry function of transient receptor potential canonical (TRPC) 1 and 4 channels in endothelial cells. Mol. Pharmacol. 81, 510-526. doi: 10.1124/mol.111.074658

Suresh, K., Servinsky, L., Reyes, J., Baksh, S., Undem, C., Caterina, M., et al. (2015). Hydrogen peroxide-induced calcium influx in lung microvascular endothelial cells involves TRPV4. Am. J. Physiol. Cell. Mol. Physiol. 309, L1467-L1477. doi: 10.1152/ajplung.00275.2015

Tauseef, M., Farazuddin, M., Sukriti, S., Rajput, C., Meyer, J. O., Ramasamy, S. K., et al. (2016). Transient receptor potential channel 1 maintains adherens junction plasticity by suppressing sphingosine kinase 1 expression to induce endothelial hyperpermeability. FASEB J. 30, 102-110. doi: 10.1096/fj.15-275891

Thakore, P., and Earley, S. (2019). Transient receptor potential channels and endothelial cell calcium signaling. Comp. Physiol. 9, 1249-1277. doi: 10.1002/ cphy.c180034

Thomas, K. C., Roberts, J. K., Deering-Rice, C. E., Romero, E. G., Dull, R. O., Lee, J., et al. (2012). Contributions of TRPV1, endovanilloids, and endoplasmic reticulum stress in lung cell death in vitro and lung injury. Am. J. Physiol. Cell. Mol. Physiol. 302, L111-L119. doi: 10.1152/ajplung.00231.2011

Thorneloe, K. S., Cheung, M., Bao, W., Alsaid, H., Lenhard, S., Jian, M.-Y., et al. (2012). An orally active TRPV4 channel blocker prevents and resolves pulmonary edema induced by heart failure. Sci. Transl. Med. 4:159ra148. doi: 10.1126/scitranslmed.3004276 
Tiruppathi, C., Ahmmed, G. U., Vogel, S. M., and Malik, A. B. (2006). Ca 2+ signaling, TRP channels, and endothelial permeability. Microcirculation 13, 693-708. doi: 10.1080/10739680600930347

Tiruppathi, C., Freichel, M., Vogel, S. M., Paria, B. C., Mehta, D., Flockerzi, V., et al. (2002). Impairment of store-operated Ca2+ entry in TRPC4(-/-) mice interferes with increase in lung microvascular permeability. Circ. Res. 91, 70-76. doi: 10.1161/01.res.0000023391.40106.a8

Trebak, M. (2009). STIM1/Orail, ICRAC, and endothelial SOC. Circ. Res. 104, e56-e57. doi: 10.1161/CIRCRESAHA.109.196105

Tsagareli, M. G., and Nozadze, I. (2019). An overview on transient receptor potential channels superfamily. Behav. Pharmacol. doi: 10.1097/FBP. 0000000000000524 [Epub ahead of print].

Van Nieuw Amerongen, G. P., Draijer, R., Vermeer, M. A., and Van Hinsbergh, V. W. M. (1998). Transient and prolonged increase in endothelial permeability induced by histamine and thrombin: role of protein kinases, calcium, and RhoA. Circ. Res. 83, 1115-1123. doi: 10.1161/01.RES.83.11. 1115

Villalta, P. C., Rocic, P., and Townsley, M. I. (2014). Role of MMP2 and MMP9 in TRPV4-induced lung injury. Am. J. Physiol. Lung Cell. Mol. Physiol. 307, L652-L659. doi: 10.1152/ajplung.00113.2014

Villalta, P. C., and Townsley, M. I. (2013). Transient receptor potential channels and regulation of lung endothelial permeability. Pulm. Circ. 3, 802-815. doi: 10.1086/674765

Wang, M., Ji, P., Wang, R., Zhao, L., and Xia, Z. (2012). TRPV1 agonist capsaicin attenuates lung ischemia-reperfusion injury in rabbits. J. Surg. Res. 173, 153160. doi: $10.1016 /$ j.jss.2010.08.053

Watanabe, H., Davis, J. B., Smart, D., Jerman, J. C., Smith, G. D., Hayes, P., et al. (2002). Activation of TRPV4 channels (hVRL-2/mTRP12) by phorbol derivatives. J. Biol. Chem. 277, 13569-13577. doi: 10.1074/jbc.M20006 2200

Watanabe, H., Murakami, M., Ohba, T., Takahashi, Y., and Ito, H. (2008). TRP channel and cardiovascular disease. Pharmacol. Ther. 118, 337-351. doi: 10. 1016/j.pharmthera.2008.03.008
Willette, R. N., Bao, W., Nerurkar, S., Yue, T. I., Doe, C. P., Stankus, G., et al. (2008). Systemic activation of the transient receptor potential vanilloid subtype 4 channel causes endothelial failure and circulatory collapse: Part 2. J. Pharmacol. Exp. Ther. 326, 443-452. doi: 10.1124/jpet.107.134551

Winkler, E. A., Sengillo, J. D., Bell, R. D., Wang, J., and Zlokovic, B. V. (2012). Blood-spinal cord barrier pericyte reductions contribute to increased capillary permeability. J. Cereb. Blood Flow Metab. 32, 1841-1852. doi: 10.1038/jcbfm. 2012.113

Worley, P. F., Zeng, W., Huang, G. N., Yuan, J. P., Kim, J. Y., Lee, M. G., et al. (2007). TRPC channels as STIM1-regulated store-operated channels. Cell Calcium 42, 205-211. doi: 10.1016/j.ceca.2007.03.004

Wu, S., Cioffi, E. A., Alvarez, D., Sayner, S. L., Chen, H., Cioffi, D. L., et al. (2005). Essential role of a Ca2+-selective, store-operated current (ISOC) in endothelial cell permeability: determinants of the vascular leak site. Circ. Res. 96, 856-863. doi: 10.1161/01.RES.0000163632.67282.1f

Yao, X., and Garland, C. J. (2005). Recent developments in vascular endothelial cell transient receptor potential channels. Circ. Res. 97, 853-863. doi: 10.1161/01. RES.0000187473.85419.3e

Yin, J., Hoffmann, J., Kaestle, S. M., Neye, N., Wang, L., Baeurle, J., et al. (2008). Negative-feedback loop attenuates hydrostatic lung edema via a cGMPdependent regulation of transient receptor potential vanilloid 4. Circ. Res. 102, 966-974. doi: 10.1161/CIRCRESAHA.107.168724

Conflict of Interest: The authors declare that the research was conducted in the absence of any commercial or financial relationships that could be construed as a potential conflict of interest.

Copyright (C) 2020 Genova, Gaglioti and Munaron. This is an open-access article distributed under the terms of the Creative Commons Attribution License (CC BY). The use, distribution or reproduction in other forums is permitted, provided the original author(s) and the copyright owner(s) are credited and that the original publication in this journal is cited, in accordance with accepted academic practice. No use, distribution or reproduction is permitted which does not comply with these terms. 